\title{
Identidad Social - Cultural en la formación de futuros docentes en la Universidad del Atlántico.
}

Social - Cultural Identity in the training of future teachers at the Atlantic University.

\section{Lizeth Del Carmen Salas Medina}

Magister en Educación

Especialista en Química Orgánica

Licenciada en Ciencias Naturales y Educación Ambiental Miembro del grupo de investigación Gecit

Universidad del Atlántico - Colombia

lichy.salas@hotmail.com 


\section{Resumen}

En los últimos años el gobierno colombiano ha venido impulsando planes de mejora en la educación; sin embargo, el tema de la identidad social y cultural de los docentes no ha sido suficientemente abordado por los investigadores. Una prueba de esto, es el escaso registro de estudios que a nivel nacional aborden esta temática como una situación que finalmente repercuta en todo el sistema educativo de la nación.

En el presente estudio tuvo como objetivo determinar los indicadores que inciden en la identidad social - cultural de los futuros docentes de la Universidad del Atlántico. Para ello se realizó una investigación de naturaleza descriptivacorrelacional, donde se aplicó la Escala de Identidad Social - Cultural universitaria, para investigar estos indicadores. Se seleccionó una muestra de la población de estudiantes de los programas de licenciatura de la Facultad de Educación. A partir del estudio se pudo determinar que indicadores tales como: sentido de pertenencia, manejo de la diversidad, investigador educativo y preparación para el aprendizaje, inciden fuertemente en la identidad social - cultural de los docentes en formación.

\section{Abstract}

In recent years the Colombian government has been promoting plans to improve education; however, the issue of teachers' social and cultural identity has not been sufficiently addressed by researchers. Proof of this is the limited record of studies that address this issue at the national level, as a situation that ultimately affects the entire educational system of the nation.

The objective of this study is to determine the indicators that affect the social cultural identity of future teachers of the Atlantic University. For this, a descriptivecorrelational research was carried out, where the University Social-Cultural Identity Scale was applied to investigate these indicators. A sample of the student population was selected from the bachelor's programs of the Faculty of Education. From the study, it was possible to determine that indicators such as: sense of belonging, diversity management, educational researcher and preparation for learning, strongly influence the social - cultural identity of teachers in training.

\section{Palabras Clave}

Identidad, Social-cultural, formación de docentes.

\section{Keywords}

Identity, Social-cultural, teacher training. 


\section{Introducción}

En los procesos educativos, el trabajo docente es uno de los factores esenciales para la calidad de los aprendizajes de los estudiantes tal como lo afirma Fernández (2017), quien en referencia al enfoque de derechos promovido por la UNESCO (2005), expone que se debe tener en cuenta y respetar los contextos, considerar e incluir los saberes propios de las comunidades y poblaciones, promover la inclusión, contribuir a dar sentido a los proyectos de vida de los educandos, y aportar al fortalecimiento de su identidad y autoestima, así como a la construcción de ciudadanía. En efecto, existe suficiente evidencia del valor estratégico que tiene el trabajo docente en el cumplimiento de los objetivos de los sistemas educativos. Si bien sus saberes y sus prácticas pedagógicas influyen en el quehacer educativo, también sus percepciones y actitudes hacia la profesión y las actividades relacionadas a su rol, tienen incidencia en su desempeño y en el de sus estudiantes.

En la misma línea, al hablar de futuros docentes, Seda (2012) indica que las actitudes juegan un rol muy importante en el éxito de la carrera docente dado que estas los llevan a comportarse de una manera positiva o negativa; en pocas palabras, a construir identidades sociales en el conocimiento, cultura, idiosincrasia, raza, estratos y pensamientos, lo que se somatiza directamente en el ejercicio profesional cuando entra en contacto con sus estudiantes, colegas y superiores.

Aunque las instituciones educativas han tenido procesos de cambio, no se ha tenido en cuenta la necesidad de romper con las líneas directrices que desde el génesis de los sistemas educativos se derivan de la centralización de los asuntos de la educación, la transmisión exclusiva de contenidos académicos, el carácter seleccionador e individualista, entre otros. Esto sin considerar, que para dar cumplimiento al objetivo de educar realmente en la vida y para la vida, se parte de la superación definitiva de los enfoques tecnológicos, funcionalistas, asociados a la burocracia, para abrirse a los fundamentos de corte relacional, culturalcontextual y comunitario, a partir de los cuales prime la relación entre todas las personas que trabajan dentro y fuera de la institución, donde se refleje el dinamismo social y cultural de la institución al servicio de toda la comunidad.

Desde esta perspectiva, actualmente a nivel educativo debe tener en cuenta aspectos como el contexto, aunado a la necesidad de adaptarse a las exigencias del mismo, lo cual cada día adquiere más importancia para la enseñanza y el aprendizaje; así también, la visión de la enseñanza no desde un enfoque técnico, la no transmisión de un conocimiento acabado y formal, sino más bien un conocimiento en construcción. Una visión en la que la educación es más que un compromiso para cumplir con las políticas de un país, sino que se relaciona directamente con el desarrollo de la persona como un factor importante para el conocimiento profesional. 
Por lo anterior, es de suma importancia para la docencia el aprendizaje de la relación, la convivencia, la cultura del contexto y el desarrollo de la capacidad de interacción de cada persona con el resto del grupo, con sus iguales y con la comunidad que enmarca la educación. De ahí que sea fundamental comprender el conocimiento profesional como un proceso que va más allá de una taxonomía de rasgos unificadores de toda la profesión docente.

En este contexto, se determinan los indicadores que inciden en la identidad social - cultural de los docentes que se forman en la Universidad del Atlántico, a través de sus nueve programas de licenciatura. Se realiza una investigación de naturaleza descriptiva-correlacional, donde se diseña y aplica la Escala de Identidad Social - Cultural universitaria, obteniendo información sobre los indicadores que inciden fuertemente en la identidad social - cultural de los docentes en formación.

\section{Referentes teóricos}

La creciente complejidad social de la educación (aún más en el futuro) debería provocar que la profesión docente se hiciera menos individual y más colectiva, superando el punto de vista estrictamente particular aplicado al conocimiento profesional, en el que la colaboración entre los compañeros está ausente y en la cual el profesorado se convierte en instrumento mecánico y aislado de aplicación y reproducción, con unas competencias que se limitan a la aplicación técnica en su aula.

Este ideal, supone construir un conocimiento profesional que dote a la docencia de instrumentos intelectuales que faciliten las capacidades reflexivas colectivas sobre la propia práctica docente donde la meta principal no sea otra que aprender a interpretar, comprender y reflexionar sobre la enseñanza y la realidad social de forma comunitaria. De acuerdo con esto, necesario que los docentes conozcan el valor de su identidad social y cultural, tal como lo argumentan Rivero y Martínez (2016) al señalar que "es innegable que la cultura y la identidad se presentan como términos necesarios para comprender los mundos contemporáneos, y una distinción precisa entre ambas nociones resulta imprescindible para el análisis de los procesos sociales". En esa misma línea, Gee (2000) asocia la identidad con el reconocimiento de un individuo en un contexto dado, también Durkheim (1986) expresa que "lo que convierte al hombre en una persona es aquello en que coincide con los demás miembros de su grupo social"; estas posturas resaltan la importancia para las ciencias humanas de este concepto asociado a la función social del individuo.

Así mismo, sobre el concepto de identidad, Rojas (2004) lo expone como una explicación de lo interno y su enlace con el contexto, esto evidencia que para el ser humano desde el nacimiento se establece una conexión entre el desarrollo interno y el medio ambiente. Esta relación individuo - medio ambiente, permite que la identidad se desarrolle dentro de pautas culturales e históricas, con un período evolutivo propio, con un conjunto de significaciones y representaciones que son relativamente permanentes, es decir, la identidad no es fija ni estática, 
dejando en claro que ésta puede cambiar en cualquier momento por cuenta de un proceso o varios procesos que en ello influyan.

Esta alusión al término de identidad cultural, conlleva a analizarlo desde la postura de Mendo (2014) quien la concibe como "un conjunto de creencias, modos de pensar, fines, valores, modo de percibir las cosas e incluso concepciones del mundo, que son comunes o compartidas por un conjunto de personas en un determinado lugar". Desde otra voz, pero claramente en concordancia se cita a Espinoza, Guamán y Gómez (2016), quienes aportan una visión de identidad cultural como el conjunto de elementos dentro de un grupo social que actúan para que los individuos que lo forman puedan fundamentar su sentimiento de pertenencia en respuesta a los intereses, normas y rituales que comparten dichos grupos dentro de la cultura.

La identidad cultural retoma el concepto de evolución cultural, pues el ser humano en construcción de su identidad asimila como propios los cambios como la conformación de ciudades y organizaciones en la defensa mutua de sus bienes y de sus personas, donde el bien común para a un segundo plano para dar paso a un todo solidario. En este sentido, Iñiguez (2001) se refiere a la identidad en el contexto social como “... una identificación con quienes nos rodean como una diferenciación estricta respecto de ellos y ellas" (p.209). Sobre este respecto, Molano (2007) opina que para poder comprenderla es imprescindible conocer la evolución del concepto de cultura y cómo ha llegado hasta los días actuales.

En resumen, se entiende la identidad cultural como el conglomerado de valores, tradiciones, símbolos, creencias y modos de comportamiento que cohesionan la pertenencia de un individuo a un determinado grupo social, que actúan para que éstos puedan fundamentar su sentimiento de ser parte del mismo, que validan su pertenencia, tal como lo expresan Briones (2007), Espinoza, Guamán y Gómez (2016), para quienes las identidades son prescriptivas de una manera de comportarse en respuesta a los intereses, códigos, normas y rituales que comparten dichos grupos dentro de la cultura dominante.

Cuando hay carencia de esta identidad, el individuo es fácilmente vulnerable a la pérdida de las costumbres del grupo en el que fue criado, y en esa medida es libre para adoptar o imitar los comportamientos de la cultura con la que tiene apego, tanto en su modo de hablar, como en su vestimenta y hasta en su forma de pensar. Este fenómeno es abordado por Paqui y Poma (2013) como la pérdida de las características que distinguen al ser humano de otros, aquellos comportamientos que indican quienes son y a qué comunidad pertenecen, involucra el entorno, la historia, los valores, la idiosincrasia, etc. La cuestión es esta: la identidad cultural depende su existencia del reconocimiento y valoración del sujeto, implica valorarse como integrantes de forma personal y a los demás miembros. Una de las consecuencias de este fenómeno es la paulatina desaparición de las costumbres y tradiciones, la pérdida de la identidad cultural se manifiesta en la impostación de otra identidad que inicia con la identificación con el otro, proceso en el que se desplazan los valores autóctonos por unos "prestados", aun cuando cada cultura es única y debe ser respetada y valorada como tal. 
Como se ha analizado, la identidad resulta de un complejo proceso psicosocial en el cual los aspectos que caracterizan a un grupo se internalizan en el ser humano hasta adherirse como su esencia y convertirse en su marca distintiva de otros grupos sociales. Desde ese mismo principio, pero en un proceso introspectivo, el ser humano es capaz de reconocer atributos propios que lo diferencian de los miembros del grupo de referencia, reafirmando lo que sustenta Iñiguez (2001) "[...] una identificación con quienes nos rodean como una diferenciación estricta respecto de ellos y ellas" (p.209).

Por último, los fenómenos de identidad se ponen a prueba cuando este conjunto de valores se somete a un proceso de choque con otras culturas así coincidan territorial y lingüísticamente. La universidad es el universo ideal que ejemplifica este enfrentamiento de identidades, por la mezcla de miles de individuos con amplia gama de atributos que convergen en un solo ideal: educarse; y aun así con esta meta clara, el tema de identidad que ya pasa al plano profesional nuevamente se somete a juicio por la necesidad de evaluar si en la formación profesional hay un proceso de identificación de los contenidos, perfiles laborales y otros aspectos.

En esta medida, el asunto de la identidad en la universidad se analiza desde los indicadores sociales y culturales en los docentes en formación de la Universidad del Atlántico, una institución de educación superior del Caribe Colombiano con la mayor oferta de programas de licenciatura adscritos a la facultad de educación, de ahí el interés por este grupo en específico, y así cumplir con el objetivo de determinar la incidencia de estos indicadores en los procesos de identidad con respecto a su perfil ocupacional, el alma mater, aspecto académicos de los futuros docentes que actualmente cursan las diferentes licenciaturas en esta Universidad.

\section{Metodología}

Esta investigación de corte descriptivo-correlacional abordó una interpretación cuantitativa y cualitativa de la información. En este caso, se trata de analizar el asunto de la identidad en la universidad desde los indicadores sociales y culturales que se evidencian en los docentes en formación de la Universidad del Atlántico, igualmente, se propone discutir el influjo de ésta en el proceso de autoevaluación, bienestar profesional y laboral de los futuros maestros. Las características de los resultados obtenidos es posible usarlos como insumo para otras investigaciones y poblaciones, desde sus propios intereses, teniendo en cuenta las obvias particularidades, siempre y cuando se tomen con reserva las inferencias de las conclusiones.

\section{La investigación se desarrolló en tres (3) etapas:}

Inicialmente se realizó una revisión de los principales estudios sobre percepciones y actitudes docentes a través de bibliotecas, bases de datos y diálogo con otros investigadores que han abordado la temática. Luego se aplicó una Escala de Identidad Social - Cultural universitaria, cuya construcción se realizó a partir del análisis de algunos indicadores relacionados con la identidad, 
diseñado por la autora para esta investigación. Las categorías diseñadas se asocian a la vocación docente, rol del docente sobre la naturaleza interpersonal del aprendizaje de los estudiantes, avance de la práctica profesional en los futuros maestros de la universidad del Atlántico y las etapas de la formación docente de los estudiantes de la facultad de educación en la Universidad del Atlántico.

En la segunda etapa, reconocida como el trabajo de campo, se visitaron otras universidades a nivel nacional, principalmente a las públicas, adscritas a ASCOFADE del SUE Caribe (Universidad de Córdoba, Universidad de Magdalena, Universidad de Sucre, Universidad de la Guajira, Universidad de Cartagena, Universidad del Atlántico, Universidad popular del Cesar), para lograr una realimentación sobre el desarrollo del trabajo.

En la tercera etapa, se analizó la información levantada a partir de la aplicación del instrumento teniendo en cuenta los aspectos presentados en cada una de las categorías. Complementariamente para aportar integralidad al proceso investigativo, se propuso realimentar estos resultados con la decanatura de la Facultad de Educación, como sustento del proceso de autoevaluación institucional.

\section{Población y muestra}

El estudio se realizó con la participación de estudiantes de los nueve (9) programas de licenciatura adscritos a la Facultad de Educación de la Universidad del Atlántico: Humanidades y Lengua Castellana, Educación Infantil, Educación Física, Ciencias Naturales, Educación Especial, Educación Artística, Lenguas Extranjeras, Ciencias Sociales, y Matemáticas.

La población estuvo constituida por 7299 estudiantes, de los cuales se conformó una muestra de 365 estudiantes a partir de la sumatoria de nueve muestras de cada uno de los programas de licenciatura existentes, para ello se consideró un muestreo aleatorio estratificado con afijación proporcional, teniendo en cuenta un nivel de confianza del 95\%.

\section{Técnicas e instrumentos}

El instrumento aplicado a estudiantes de los programas de licenciatura (primer periodo del año 2019), fue una Escala de Identidad Social - Cultural Universitaria diseñada para este estudio, el cual es un instrumento de 36 reactivos dicotómicos donde se establecen dos variables: 1. Identidad Social Cultural. 2. Formación docente; cuatro dimensiones: 1. Vocación Docente, 2. Rol del Docente sobre la Naturaleza Interpersonal del Aprendizaje, 3. Avance de la Práctica Profesional, 4. Etapas de la Formación Docente; y en cada dimensión tres indicadores, realizándose por cada uno, tres afirmaciones.

La investigación optó por el diseño de la escala de identidad social- cultural universitaria, debido a que en la primera fase de este estudio se encontró que no existían cuestionarios de esta índole, específicamente se encontraron escalas que buscan determinar el grado de identidad institucional pero no desde la óptica de 
lo sociocultural y mucho menos desde la formación docente. La información fue procesada en el programa estadístico SPSS versión 25.0.

\section{Resultados y Discusión}

La presentación de los resultados y su discusión se realiza por dimensiones con sus respectivos indicadores. Los datos que se presentan en esta primera parte corresponden a la dimensión vocación docente que en el instrumento aborda los indicadores: sentido de pertenencia, relaciones interpersonales y manejo de la diversidad cada uno con tres ítems, para un total de nueve.

Tabla 1. Resultados de la dimensión vocación docente para el indicador sentido de pertenencia

\begin{tabular}{|lll|l|l|l|l|l|}
\cline { 3 - 8 } \multicolumn{1}{c|}{} & \multicolumn{2}{c|}{ Item 1 } & \multicolumn{2}{c|}{ Item 2 } & \multicolumn{2}{c|}{ Item 3 } \\
\cline { 3 - 9 } \multicolumn{1}{c|}{} & $\mathrm{F}$ & $\%$ & $\mathrm{f}$ & $\%$ & $\mathrm{f}$ & $\%$ \\
\hline Totalmente de acuerdo & 181 & 49,6 & 143 & 39,2 & 129 & 35,3 \\
\hline De acuerdo & 168 & 46,0 & 168 & 46,0 & 177 & 48,5 \\
\hline $\begin{array}{l}\text { Ni en acuerdo ni en } \\
\text { desacuerdo }\end{array}$ & 12 & 3,3 & 48 & 13,2 & 56 & 15,3 \\
\hline En desacuerdo & 4 & 1,1 & 6 & 1,6 & 3 & 0,8 \\
\hline Total & $\mathbf{3 6 5}$ & 100,0 & $\mathbf{3 6 5}$ & 100,0 & 365 & 100,0 \\
\hline
\end{tabular}

Fuente(s): Salas (2018).

Tabla 2. Resultados de la dimensión vocación docente para el indicador relaciones interpersonales

\begin{tabular}{|l|l|l|l|l|l|l|}
\cline { 2 - 7 } \multicolumn{1}{c|}{} & \multicolumn{2}{c|}{ Item 4 } & \multicolumn{2}{c|}{ Item 5 } & \multicolumn{2}{c|}{ Item 6 } \\
\cline { 2 - 8 } \multicolumn{1}{c|}{} & $\mathrm{F}$ & $\%$ & $\mathrm{f}$ & $\%$ & $\mathrm{f}$ & $\%$ \\
\hline Totalmente de acuerdo & 95 & 26,0 & 67 & 18,4 & 107 & 29,3 \\
\hline De acuerdo & 195 & 53,4 & 198 & 54,2 & 170 & 46,6 \\
\hline Ni en acuerdo ni en desacuerdo & 61 & 16,7 & 75 & 20,5 & 74 & 20,3 \\
\hline En desacuerdo & 11 & 3,0 & 17 & 4,7 & 9 & 2,5 \\
\hline Totalmente en desacuerdo & 3 & 0,8 & 8 & 2,2 & 5 & 1,4 \\
\hline Total & 365 & 100,0 & 365 & 100,0 & 365 & 100,0 \\
\hline
\end{tabular}

Fuente(s): Salas (2018).

Tabla 3. Resultados de la dimensión vocación docente para el indicador Manejo de la diversidad

\begin{tabular}{|l|l|l|l|l|l|l|}
\cline { 2 - 8 } \multicolumn{1}{c|}{} & \multicolumn{2}{c|}{ Item 7 } & \multicolumn{2}{c|}{ Item 8 } & \multicolumn{2}{c|}{ Item 9 } \\
\cline { 2 - 8 } \multicolumn{1}{c|}{} & $\mathrm{F}$ & $\%$ & $\mathrm{f}$ & $\%$ & $\mathrm{~F}$ & $\%$ \\
\hline Totalmente de acuerdo & 86 & 23,6 & 92 & 25,2 & 95 & 26,0 \\
\hline De acuerdo & 170 & 46,6 & 187 & 51,2 & 16 & 4,4 \\
\hline Ni en acuerdo ni en desacuerdo & 74 & 20,3 & 64 & 17,5 & 66 & 18,1 \\
\hline En desacuerdo & 19 & 5,2 & 13 & 3,6 & 177 & 48,5 \\
\hline Totalmente en desacuerdo & 16 & 4,4 & 9 & 2,5 & 11 & 3,0 \\
\hline Total & 365 & 100,0 & 365 & 100,0 & 365 & 100,0 \\
\hline
\end{tabular}

Fuente(s): Salas (2018).

A partir de lo anterior, se resaltan los resultados de la primera dimensión y sus respectivos indicadores. Específicamente en el indicador Sentido de pertenencia, el cual Serón (2012) lo describe como la adscripción a un grupo social, la mayoría de los informantes están totalmente de acuerdo en formar parte de la universidad; mientras que para los ítems 2 y 3 opinaron estar de acuerdo. Es decir, su selección baja una alternativa con relación a sentirse satisfecho de pertenecer a la 
Universidad del Atlántico y a valorar el papel que desempeña el docente en formación en la universidad.

En este sentido, Larrosa (2010), manifiesta que, desde el punto de vista de las transformaciones sociales, el cambio operado en el concepto de vocación se evidencia en el incremento de las exigencias de la familia y de la sociedad con el profesorado. Estos cambios también exigen del profesorado nuevas competencias que le permitan abordar la multiplicidad de situaciones no previsibles que diariamente se le presentan en el desarrollo de sus funciones y la toma de decisiones con autonomía.

En cuanto al indicador relaciones interpersonales, la mayoría eligió como alternativa de respuesta: de acuerdo. Es decir, está de acuerdo en que se transmite conocimientos socializado sobre el rescate de la identidad social cultural, socializa con el profesorado estrategias para el desempeño de valores socio-culturales y en estimular la participación entre todos los actores involucrados en el proceso educativo. Al respecto González (1999), afirma que “...es imposible concebir al ser humano desculturalizado, o mejor, desconectado de su medio natural, cultural y social; ... y una parte del desarrollo de los individuos (normalmente ligado a la cultura en la que ese individuo vive) sólo puede asegurarse desde la intervención escolar" (p. 227).

Sobre los resultados para el indicador manejo de la diversidad es posible percibir que la mayoría eligió como alternativa de respuesta: de acuerdo para los ítems 7 y 8 . Es decir, están de acuerdo en que interviene en las decisiones de las actividades de interés para todos y en que promueve un sentimiento de pertenecía en la comunidad estudiantil. Sin embargo, la mayoría está en desacuerdo en que utiliza la diversidad cultural para fortalecer el manejo cultura institucional.

Sobre este asunto, Mercado y Hernández (2010) opinan que, en la sociedad moderna, los sujetos se adscriben a una diversidad de grupos, razón por la cual se habla de identidad étnica, religiosa, política, laboral, profesional, de género, entre otras. La pertenencia a varios grupos provoca que los sujetos lleven a cabo un proceso de selección de valores, creencias, informaciones, opiniones, actitudes, prácticas y símbolos, con los cuales se definen a sí mismos, explican la realidad y guían sus acciones. De ahí que la identidad social y cultural implica un "proceso de construcción del sentido atendiendo a un atributo cultural, o un conjunto relacionado de atributos culturales, al que se da prioridad sobre el resto de las fuentes de sentido" (Castells, 1999, p.28).

En lo que respecta a la dimensión Rol del Docente sobre la Naturaleza Interpersonal del Aprendizaje, el instrumento presenta en un orden ascendente 9 afirmaciones más que van del número diez a la dieciocho, donde se dirimieron algunos indicadores como: Naturaleza Interpersonal del Aprendizaje y transmisor de conocimientos, guía del proceso de Aprendizaje, investigador educativo: 
Tabla 4. Resultados de la dimensión Rol del docente sobre la Naturaleza Interpersonal del Aprendizaje y el indicador transmisor de conocimientos

\begin{tabular}{|l|l|l|l|l|l|l|}
\cline { 2 - 8 } \multicolumn{1}{c|}{} & \multicolumn{2}{c|}{ Item 10 } & \multicolumn{2}{c|}{ Item 11 } & \multicolumn{2}{c|}{ Item 12 } \\
\cline { 2 - 8 } \multicolumn{1}{c|}{} & $\mathrm{F}$ & $\%$ & $\mathrm{f}$ & $\%$ & $\mathrm{~F}$ & $\%$ \\
\hline Totalmente de acuerdo & 110 & 30,1 & 138 & 37,8 & 115 & 31,5 \\
\hline De acuerdo & 189 & 51,8 & 165 & 45,2 & 176 & 48,2 \\
\hline Ni en acuerdo ni en desacuerdo & 52 & 14,2 & 46 & 12,6 & 56 & 15,3 \\
\hline En desacuerdo & 8 & 2,2 & 12 & 3,3 & 9 & 2,5 \\
\hline Totalmente en desacuerdo & 6 & 1,6 & 4 & 1,1 & 9 & 2,5 \\
\hline Total & 365 & 100,0 & 365 & 100,0 & 365 & 100,0 \\
\hline
\end{tabular}

Fuente(s): Salas (2018).

Tabla 5. Resultados de la dimensión Rol del docente sobre la Naturaleza Interpersonal del Aprendizaje y el indicador guía del proceso de Aprendizaje

Fuente(s): Salas (2018).

\begin{tabular}{|l|l|l|l|l|l|l|}
\cline { 2 - 7 } \multicolumn{1}{c|}{} & \multicolumn{2}{c|}{ Item 13 } & \multicolumn{2}{c|}{ Item 14 } & \multicolumn{2}{c|}{ Item 15 } \\
\cline { 2 - 8 } \multicolumn{1}{c|}{} & $\mathrm{F}$ & $\%$ & $\mathrm{f}$ & $\%$ & $\mathrm{f}$ & $\%$ \\
\hline Totalmente de acuerdo & 153 & 41,9 & 118 & 32,3 & 111 & 30,4 \\
\hline De acuerdo & 157 & 43,0 & 165 & 45,2 & 182 & 49,9 \\
\hline Ni en acuerdo ni en desacuerdo & 40 & 11,0 & 60 & 16,4 & 53 & 14,5 \\
\hline En desacuerdo & 7 & 1,9 & 18 & 4,9 & 17 & 4,7 \\
\hline Totalmente en desacuerdo & 8 & 2,2 & 4 & 1,1 & 2 & 0,5 \\
\hline Total & 365 & 100,0 & 365 & 100,0 & 365 & 100,0 \\
\hline
\end{tabular}

Tabla 6. Resultados de la dimensión Rol del docente sobre la Naturaleza Interpersonal del Aprendizaje y el indicador investigador educativo

\begin{tabular}{|l|l|l|l|l|l|l|}
\cline { 2 - 8 } \multicolumn{1}{c|}{} & \multicolumn{2}{c|}{ Item 16 } & \multicolumn{2}{c|}{ Item 17 } & \multicolumn{2}{c|}{ Item 18 } \\
\cline { 2 - 8 } \multicolumn{1}{c|}{} & $\mathrm{f}$ & $\%$ & $\mathrm{f}$ & $\%$ & $\mathrm{f}$ & $\%$ \\
\hline Totalmente de acuerdo & 84 & 23,0 & 90 & 24,7 & 127 & 34,8 \\
\hline De acuerdo & 4 & 1,1 & 6 & 1,6 & 1 & 0,3 \\
\hline Ni en acuerdo ni en desacuerdo & 55 & 15,1 & 67 & 18,4 & 60 & 16,4 \\
\hline En desacuerdo & 18 & 4,9 & 13 & 3,6 & 17 & 4,7 \\
\hline Totalmente en desacuerdo & 204 & 55,9 & 189 & 51,8 & 160 & 43,8 \\
\hline Total & $\mathbf{3 6 5}$ & 100,0 & $\mathbf{3 6 5}$ & $\mathbf{1 0 0 , 0}$ & $\mathbf{3 6 5}$ & $\mathbf{1 0 0 , 0}$ \\
\hline
\end{tabular}

Fuente(s): Salas (2018).

Tal como puede evidenciarse en las tablas anteriores, en el primer indicador Naturaleza Interpersonal del Aprendizaje y transmisor de conocimientos, la mayoría eligió la opción de respuesta: de acuerdo. Esto es, que los estudiantes manifestaron estar de acuerdo con promover la representación de ideas esenciales a través de la escritura espontanea, considerar las estrategias de clase como elemento necesario para organizar las actividades escolares y con trasmitir el conocimiento adquirido con sus compañeros de clase.

En relación con el indicador guía del proceso de Aprendizaje, puede notarse que la mayoría de los encuestados escogió estar de acuerdo con motivar a sus compañeros de clase para que realicen el trabajo en equipo lo mejor posible, dedica tiempo para comentar los logros alcanzados con otros estudiantes y con lograr alcanzar todas las actividades planificadas por el profesor en el salón de clases. Cuando se indagó sobre el indicador investigador educativo es evidente que la mayoría de los encuestados escogió estar totalmente en desacuerdo; lo que indica que ellos sienten que no promueven el adelanto de la ciencia para buscar soluciones a los problemas de la sociedad, ni desarrollan avances en las diferentes áreas del conocimiento para responder a los retos presentes del mundo actual, así como tampoco han adquirido competencias investigativas durante el desarrollo de las actividades programadas. 
Por otra parte, los resultados de la dimensión Avance de la Práctica Profesional indican la opinión de los informantes sobre indicadores como: apropiación del marco legal, desarrollo del plan de estudio, mediación del Aprendizaje así:

Tabla 7. Resultados de la dimensión avance de la Práctica Profesional, para el indicador apropiación del marco legal

\begin{tabular}{|l|l|l|l|l|l|l|}
\cline { 2 - 8 } \multicolumn{1}{c|}{} & \multicolumn{2}{c|}{ Item 19 } & \multicolumn{2}{c|}{ Item 20 } & \multicolumn{2}{c|}{ Item 21 } \\
\cline { 2 - 8 } \multicolumn{1}{c|}{} & $\mathrm{f}$ & $\%$ & $\mathrm{~F}$ & $\%$ & $\mathrm{f}$ & $\%$ \\
\hline Totalmente de acuerdo & 123 & 33,7 & 142 & 38,9 & 93 & 25,5 \\
\hline De acuerdo & 156 & 42,7 & 162 & 44,4 & 173 & 47,4 \\
\hline Ni en acuerdo ni en desacuerdo & 73 & 20,0 & 49 & 13,4 & 65 & 17,8 \\
\hline En desacuerdo & 9 & 2,5 & 7 & 1,9 & 22 & 6,0 \\
\hline Totalmente en desacuerdo & 4 & 1,1 & 5 & 1,4 & 12 & 3,3 \\
\hline Total & 365 & 100,0 & 365 & 100,0 & 365 & 100,0 \\
\hline
\end{tabular}

Fuente(s): Salas (2018).

Tabla 8. Resultados de la dimensión avance de la Práctica Profesional, para el indicador desarrollo del plan de estudio

\begin{tabular}{|l|l|l|l|l|l|l|}
\cline { 2 - 8 } \multicolumn{1}{c|}{} & \multicolumn{2}{c|}{ Item 22 } & \multicolumn{2}{c|}{ Item 23 } & \multicolumn{2}{c|}{ Item 24 } \\
\cline { 2 - 8 } \multicolumn{1}{c|}{} & $\mathrm{f}$ & $\%$ & $\mathrm{~F}$ & $\%$ & $\mathrm{~F}$ & $\%$ \\
\hline Totalmente de acuerdo & 111 & 30,4 & 78 & 21,4 & 93 & 25,5 \\
\hline De acuerdo & 177 & 48,5 & 193 & 52,9 & 211 & 57,8 \\
\hline Ni en acuerdo ni en desacuerdo & 51 & 14,0 & 65 & 17,8 & 38 & 10,4 \\
\hline En desacuerdo & 17 & 4,7 & 23 & 6,3 & 15 & 4,1 \\
\hline Totalmente en desacuerdo & 9 & 2,5 & 6 & 1,6 & 8 & 2,2 \\
\hline Total & 365 & 100,0 & 365 & 100,0 & 365 & 100,0 \\
\hline
\end{tabular}

Fuente(s): Salas (2018).

Tabla 9. Resultados de la dimensión avance de la Práctica Profesional, para el indicador mediación del Aprendizaje

\begin{tabular}{|l|l|l|l|l|l|l|}
\cline { 2 - 8 } \multicolumn{1}{c|}{} & \multicolumn{2}{c|}{ Item 25 } & \multicolumn{2}{c|}{ Item 26 } & \multicolumn{2}{c|}{ Item 27 } \\
\cline { 2 - 8 } \multicolumn{1}{c|}{} & $\mathrm{f}$ & $\%$ & $\mathrm{~F}$ & $\%$ & $\mathrm{~F}$ & $\%$ \\
\hline Totalmente de acuerdo & 97 & 26,6 & 103 & 28,2 & 145 & 39,7 \\
\hline De acuerdo & 215 & 58,9 & 217 & 59,5 & 169 & 46,3 \\
\hline Ni en acuerdo ni en desacuerdo & 40 & 11,0 & 31 & 8,5 & 29 & 7,9 \\
\hline En desacuerdo & 5 & 1,4 & 7 & 1,9 & 16 & 4,4 \\
\hline Totalmente en desacuerdo & 8 & 2,2 & 7 & 1,9 & 6 & 1,6 \\
\hline Total & 365 & 100,0 & 365 & 100,0 & 365 & 100,0 \\
\hline
\end{tabular}

Fuente(s): Salas (2018).

Los resultados evidencian que la mayoría de los encuestados escogió estar de acuerdo para el indicador apropiación del marco legal; puesto que la mayoría considera que cumplen con las políticas educativas universitarias establecidas en el marco legal, reconoce la educación como un derecho público que puede ser prestado por el Estado colombiano y considera que el sistema de educación superior responde a las necesidades de la sociedad. Seguidamente, el análisis de los resultados para el indicador desarrollo del plan de estudio expone que los encuestados escogieron estar de acuerdo en concebir el plan de estudio a la educación como un instrumento de igualdad social, en la respuesta de la universidad a las necesidades de las comunidades de adaptarse a cambios en el entorno social y en que desarrolla sus competencias en el marco de una sociedad con igualdad de oportunidades para todos. Al indagar sobre el indicador mediación del Aprendizaje, es posible verificar que la mayoría de los encuestados coincide en que formar parte en el proceso de aprendizaje de representación 
activa, proporciona el compromiso de un ser humano en el proceso de aprendizaje para la transformación de la realidad y permite la interacción entre sus compañeros de clase y su realidad.

Por último, se presentan los resultados para esta dimensión Etapas de la Formación Docente donde se tuvieron en cuenta los indicadores: preparación para el Aprendizaje, introducción de nuevos contenidos, transferencia del conocimiento:

Tabla 10. Resultados de la dimensión Etapas de la Formación Docente y el indicador preparación para el Aprendizaje.

\begin{tabular}{|l|l|l|l|l|l|l|}
\cline { 2 - 8 } \multicolumn{1}{c|}{} & \multicolumn{2}{c|}{ Item 28 } & \multicolumn{2}{c|}{ Item 29 } & \multicolumn{2}{c|}{ Item 30 } \\
\cline { 2 - 8 } \multicolumn{1}{c|}{} & $\mathrm{f}$ & $\%$ & $\mathrm{~F}$ & $\%$ & $\mathrm{~F}$ & $\%$ \\
\hline Totalmente de acuerdo & 113 & 31,0 & 100 & 27,4 & 91 & 24,9 \\
\hline De acuerdo & 2 & 0,5 & 2 & 0,5 & 8 & 2,2 \\
\hline Ni en acuerdo ni en desacuerdo & 54 & 14,8 & 57 & 15,6 & 62 & 17,0 \\
\hline En desacuerdo & 12 & 3,3 & 8 & 2,2 & 9 & 2,5 \\
\hline Totalmente en desacuerdo & 184 & 50,4 & 198 & 54,2 & 195 & 53,4 \\
\hline Total & $\mathbf{3 6 5}$ & $\mathbf{1 0 0 , 0}$ & $\mathbf{3 6 5}$ & 100,0 & $\mathbf{3 6 5}$ & 100,0 \\
\hline
\end{tabular}

Fuente(s): Salas (2018).

Tabla 11. Resultados de la dimensión Etapas de la Formación Docente y el indicador introducción de nuevos contenidos.

\begin{tabular}{|l|l|l|l|l|l|l|}
\cline { 2 - 8 } \multicolumn{1}{c|}{} & \multicolumn{2}{c|}{ Item 31 } & \multicolumn{2}{c|}{ Item 32 } & \multicolumn{2}{c|}{ Item 33 } \\
\cline { 2 - 8 } \multicolumn{1}{c|}{} & $\mathrm{f}$ & $\%$ & $\mathrm{~F}$ & $\%$ & $\mathrm{f}$ & $\%$ \\
\hline Totalmente de acuerdo & 106 & 29,0 & 118 & 32,3 & 109 & 29,9 \\
\hline De acuerdo & 201 & 55,1 & 181 & 49,6 & 195 & 53,4 \\
\hline Ni en acuerdo ni en desacuerdo & 42 & 11,5 & 53 & 14,5 & 45 & 12,3 \\
\hline En desacuerdo & 10 & 2,7 & 12 & 3,3 & 9 & 2,5 \\
\hline Totalmente en desacuerdo & 6 & 1,6 & 1 & 0,3 & 7 & 1,9 \\
\hline Total & 365 & 100,0 & 365 & 100,0 & 365 & 100,0 \\
\hline
\end{tabular}

Fuente(s): Salas (2018).

Tabla 12. Resultados de la dimensión Etapas de la Formación Docente y el indicador transferencia del conocimiento.

\begin{tabular}{|l|l|l|l|l|l|l|}
\cline { 2 - 7 } \multicolumn{1}{c|}{} & \multicolumn{2}{c|}{ Item 34 } & \multicolumn{2}{c|}{ Item 35 } & \multicolumn{2}{c|}{ Item 36 } \\
\cline { 2 - 8 } \multicolumn{1}{c|}{} & $\mathrm{f}$ & $\%$ & $\mathrm{~F}$ & $\%$ & $\mathrm{f}$ & $\%$ \\
\hline Totalmente de acuerdo & 119 & 32,6 & 147 & 40,3 & 111 & 30,4 \\
\hline De acuerdo & 192 & 52,6 & 163 & 44,7 & 189 & 51,8 \\
\hline Ni en acuerdo ni en desacuerdo & 43 & 11,8 & 37 & 10,1 & 37 & 10,1 \\
\hline En desacuerdo & 7 & 1,9 & 11 & 3,0 & 17 & 4,7 \\
\hline Totalmente en desacuerdo & 4 & 1,1 & 7 & 1,9 & 11 & 3,0 \\
\hline Total & $\mathbf{3 6 5}$ & $\mathbf{1 0 0 , 0}$ & $\mathbf{3 6 5}$ & 100,0 & $\mathbf{3 6 5}$ & 100,0 \\
\hline
\end{tabular}

Fuente(s): Salas (2018).

Los resultados obtenidos en el indicador preparación para el Aprendizaje, muestran que la mayoría de los encuestados escogió estar totalmente en desacuerdo, lo que indica que a ellos no se les realiza un diagnóstico para detectar las necesidades educativas de los docentes en formación, tampoco se toma en cuenta en el diagnóstico las potencialidades de los educandos, ni se toma la iniciativa de llevar una ficha historial en seguimiento del desempeño escolar.

Con respecto al indicador introducción de nuevos contenidos, se evidencia que la mayoría de los encuestados escogió estar de acuerdo con lo preguntado, es decir, con adoptar decisiones curriculares adecuadas a la realidad de los docentes en formación, desarrollar estrategias ajustadas a las necesidades educativas, y 
con integrar en la planificación contenidos relacionados a la identidad social cultural. Más adelante al revisar los resultados del indicador transferencia del conocimiento, la mayoría de los encuestados manifiestan estar de acuerdo con el hecho de desempeñar un papel significativo en la mejora de la calidad de vida, realizar prácticas profesionales generando aportes en mejoras sociales y con contribuir al desarrollo económico transfiriendo conocimiento a la ciudadanía a través de una sociedad en procesos.

Por otra parte, con el objetivo de facilitar el análisis, se han considerado unos baremos por programa, los cuales permiten visualizar y determinar niveles o grados en las diferentes afirmaciones que miden satisfacción o apreciación favorable en cada uno de los indicadores. Es de anotar que los baremos son distintos, pues por cada programa se consideraron diferentes tamaños muestrales. A menor valor corresponde mayor grado de satisfacción o de apreciación favorable.

Tabla 13. Baremo de los indicadores para cada Licenciatura de la Facultad de Ciencias de la Educación.

\begin{tabular}{|c|c|c|c|c|c|c|c|c|}
\hline $\begin{array}{c}\text { Nivel o } \\
\text { grado }\end{array}$ & Especial & $\begin{array}{c}\text { Matemá-ticas/ } \\
\text { Biología y } \\
\text { Química }\end{array}$ & Artística & Infantil & $\begin{array}{c}\text { Humanidades } \\
\text { y lengua } \\
\text { Castellana }\end{array}$ & $\begin{array}{c}\text { Ciencias } \\
\text { Sociales }\end{array}$ & $\begin{array}{c}\text { Educación } \\
\text { Física }\end{array}$ & $\begin{array}{c}\text { Idiomas } \\
\text { Extranjeros }\end{array}$ \\
\hline $\begin{array}{c}\text { Muy } \\
\text { alto }\end{array}$ & $21-42$ & $46-92$ & $13-26$ & $26-52$ & $49-98$ & $37-74$ & $74-148$ & $53-106$ \\
\hline Alto & $43-64$ & $93-139$ & $27-40$ & $53-79$ & $99-148$ & $75-112$ & $149-223$ & $107-160$ \\
\hline Medio & $65-86$ & $140-186$ & $41-44$ & $80-106$ & $149-198$ & $113-150$ & $224-298$ & $161-214$ \\
\hline Bajo & $87-108$ & $187-233$ & $45-58$ & $107-133$ & $199-248$ & $151-188$ & $299-373$ & $215-268$ \\
\hline
\end{tabular}

Fuente(s): Salas (2018).

En la tabla 13 se muestran las puntuaciones que expresan el nivel o grado de satisfacción por cada programa de las Licenciaturas en Educación. De las 36 afirmaciones planteadas, se tiene que solo las respuestas a los reactivos: 9,16 , $17,18,28,29$ y 30 fueron diferentes, en éstas se obtuvo un grado de satisfacción diferente, ya que los altos puntajes obtenidos denotan un bajo grado de satisfacción, como se muestra a continuación:

Tabla 14. Puntuaciones del nivel o grado de satisfacción por programa.

Fuente(s): Salas (2018).

\begin{tabular}{|c|c|c|c|c|c|c|c|}
\hline Programa & P9 & P16 & P17 & P18 & P28 & P29 & P30 \\
\hline Lic. Educación Especial & 90 & 92 & 98 & 99 & 71 & 70 & 70 \\
\hline Lic. Biología y Química & 205 & 72 & 78 & 43 & 157 & 150 & 160 \\
\hline Lic. Educación Artística & 18 & 52 & 54 & 57 & 44 & 40 & 50 \\
\hline Lic. Educación Infantil & 111 & 119 & 125 & 122 & 84 & 87 & 84 \\
\hline Lic. Matemáticas & 213 & 228 & 205 & 236 & 130 & 146 & 161 \\
\hline Lic. Humanidades y Lengua Castellana & 223 & 235 & 255 & 238 & 189 & 168 & 165 \\
\hline Lic. Ciencias Sociales & 176 & 164 & 172 & 182 & 88 & 118 & 140 \\
\hline Lic. Educación Física & 343 & 345 & 349 & 321 & 78 & 81 & 76 \\
\hline Lic. Idiomas Extranjeros & 252 & 253 & 206 & 236 & 191 & 180 & 156 \\
\hline
\end{tabular}

A partir de las respuestas dadas al resto de afirmaciones, se plantea que tienen altos grado de satisfacción, lo que se traduce en afirmar que poseen sentido de pertenencia hacia la universidad y la carrera profesional que eligieron cursar, lo cual se manifiesta en las buenas relaciones interpersonales que mantienen con sus pares. Consideran las estrategias de clase como elemento 
didáctico, realizan todas las actividades propuestas por el profesor, tienen apropiación del marco legal universitario, entre otras.

La afirmación 9 utiliza la diversidad cultural para fortalecer el manejo cultural institucional; en esta se obtuvo que todos los programas de licenciatura con excepción de educación artística presentan un bajo grado de satisfacción, lo que denota que a la mayoría de los estudiantes les cuesta hacer uso de la riqueza de culturas que tiene la Universidad del Atlántico, cosa distinta a lo que sucede con los estudiantes de educación artística, los cuales quizás por el ámbito y los fines de sus carrera exploran mucho más esta dimensión.

Las afirmaciones 16, 17 y 18, correspondientes al indicador investigador educativo, enmarcadas dentro de indicador del rol científico de la profesión docente, muestran a través de los altos puntajes obtenidos la poca adquisición de las competencias investigativas tan oportunas y necesarias en el educador de este siglo. Se destaca dentro de los resultados obtenidos el programa de licenciatura en Biología y Química, en el cual al parecer si se hace uso de investigación educativa como estrategia de formación del docente. Por su parte, en las afirmaciones 28, 29 y 30 que corresponden al indicador preparación para el aprendizaje, se encontró que los estudiantes del programa de educación física muestran gran manejo del proceso de formación del estudiante, ya que, según sus respuestas realizan diagnósticos de entrada con sus estudiantes y toman en cuenta todas las dimensiones del ser humano a la hora de formar. Situación diferente se tiene con los estudiantes de los otros programas donde el grado de satisfacción frente a estas afirmaciones fue bajo.

\section{Correlaciones}

Teniendo en cuenta que el nivel de medición de las variables es cualitativo ordinal, se utiliza el coeficiente de Correlación de Spearman para analizar posibles asociaciones entre éstas. Se analizan todas las posibles correlaciones entre las variables encontrando correlación estadística significativa entre las variables P9 y P29 que miden los aspectos: manejo de la diversidad y del diagnóstico académico estudiantil. En la tabla 15 se muestran los resultados:

Tabla 15. Resultados de la correlación

\begin{tabular}{|c|c|c|c|c|}
\hline & & & P9 & P29 \\
\hline \multirow{6}{*}{$\begin{array}{c}\text { Rho de } \\
\text { Spearman }\end{array}$} & \multirow{3}{*}{ P9 } & Coeficiente de correlación & 1,000 & ,108* \\
\hline & & Sig. (bilateral) & & ,040 \\
\hline & & $\mathrm{N}$ & 365 & 365 \\
\hline & \multirow{3}{*}{$\begin{array}{c}\mathrm{P} 2 \\
9\end{array}$} & Coeficiente de correlación & $108^{*}$ & 1,000 \\
\hline & & Sig. (bilateral) & ,040 & \\
\hline & & $\mathrm{N}$ & 365 & 365 \\
\hline
\end{tabular}

El p-valor asociado al coeficiente de correlación es 0,04, valor menor al nivel de significancia fijado en la investigación, lo que lleva al rechazo de la hipótesis nula de no correlación entre las variables. Aunque el valor del coeficiente de correlación no es muy alto $(0,108)$, se acepta, pues es bien sabido que en las Ciencias Sociales es difícil encontrar variables que se correlacionen altamente. 
Teniendo en cuenta que el coeficiente de correlación es positivo se concluye que a niveles altos de una variable se corresponden niveles altos en la otra y para niveles bajos en una se asocian niveles bajos en la otra. Para este caso, esto significa que cuando el docente es capaz de tomar en cuenta toda su realidad entendida como el encuentro de culturas, es idóneo para realizar una buena intervención en el escenario educativo, fruto de un asertivo diagnóstico académico de sus educandos.

\section{Conclusiones}

La identidad social - cultural no es un proceso acabado o cerrado, ya que está en permanente construcción. Es así como en la búsqueda de dicha identidad, el estudiante universitario redefine esa identidad construida con anterioridad en los primeros años de vida, pero que ahora se combina con el ejercicio profesional. En este proceso de redefinición la universidad juega un papel importante ya que a partir de cúmulo de vivencias académicas y de sucesos socioculturales que durante su paso se dan, tiene lugar a la consolidación de identidades social cultural. De acuerdo con los resultados arrojados a partir de este estudio, se concluye que efectivamente existe en el estudiantado un gran sentido de pertenencia por su universidad, tal y como lo corroboran estudios anteriores.

Sin embargo, es un sentimiento que va ligado mucho más al hecho de sentir apego por aquello que se considera propio, aquello de naturaleza material que hace sentir dueño de algo. Pero, tal como se verifica en este estudio, no sucede lo mismo cuando se habla de ese grado de identidad hacia la profesión, al ser educador. Se tiene entonces docentes en formación con poco espíritu investigador de la actividad educativa y de los fenómenos asociados a este; al igual que poco reflexivos y ahondantes de su realidad más cercana. Por el contrario, se tiene estudiantes de licenciatura con una visión de profesión docente bastante mecanicista, donde se le ve como un programa más de tipo universitario. Ahora bien, el conocer todas estas características de los docentes en formación de la Universidad del Atlántico brinda herramientas necesarias para idear estrategias que busquen desde el periodo de la formación la consecución de docentes conocedores de su labor de vida y de los desafíos a los cuales se enfrenta cada día su profesión. Se hace necesario el rescate de lo sociocultural, teniendo en cuenta que finalmente la educación es un fin social humanitario.

Referenciando los resultados obtenidos en la muestra, se puede concluir que los estudiantes de los programas de licenciatura de la Universidad del Atlántico tienen una identidad social cultural que es necesario fortalecer ya que aspectos tan importantes como el manejo de la diversidad cultural y la exploración científica de la realidad educativa se encuentran en términos generales en crisis. Igualmente cabe resaltar que estos mismos indicadores están presentes de manera positiva en algunos programas, por lo que se tendría bases de referencia para intervenir positivamente en la construcción de identidad social - cultural. 


\section{Referencias}

Briones, C. (2007). "Teorías performativas de la identidad y performatividad de las teorías". Revista Tabula Rasa, 6: 55-83.

Campos, H. (2018). "Estudio de la identidad cultural mediante una construcción epistémica del concepto identidad cultural regional». Cinta moebio 62: 199-212.

Castells, M. (1999). “El poder de la identidad”, en La era de la información. Vol. II. México: Siglo XXI.

Durkhein, E. (1986).Las formas elementales de la vida religiosa. Coloíon. México.

Espinoza, Enrique; Guamán, Verónica y Gómez, Máximo. 2016. «El continuum cultura-identidad de la comunicación y la educación ecuatoriana”. Revista Universidad y Sociedad, 8(4): 71-79.

Fernández, C. (2017). "Análisis de la calidad educativa en Andalucía desde la perspectiva del profesorado de Educación Primaria ". Tesis doctoral. Universidad de Sevilla. https://idus.us.es , handle , 01. Tesis Doctoral [Cristóbal Torres Fernández].pdf.

Gee, J. (2000). "The New Literacy Studies. From 'socially situated' to the work of the social", en The New Literacy Studies. From "socially situated" to the work of the social, editado por David Barton, Mary Hamilton y Roz Ivanič, 180-196. Nueva York: Routledge.

González, A. (1999). La formación en ámbitos no formales. En Ferreres, V. e Imbernón, F. (1999). Formación y actualización para la función pedagógica. Madrid: Síntesis.

Iñiguez, L. (2001). "Identidad: de lo personal a lo social. Un recorrido conceptual». En La constitución social de la subjetividad, editado por Crespo, E. y Soldevilla C. Madrid: Catarata.

Mendo, J. (2014). "Educación e identidad". Acceso 12 de abril de 2019. http://aulaintercultural.org/2013 /04/29/educacion-e-identidad-cultural/

Larrosa, F. (2010). Deberes y derechos del profesorado y de la comunidad educativa. Alicante: ECU.

Mercado, A. y Hernández, A. (2010). El proceso de construcción de la identidad colectiva. Convergencia, Revista de Ciencias Sociales, núm. 53, mayo - agosto 2010, pp. 229-251.

Molano, O. (2007). "Identidad cultural un concepto que evoluciona". Revista OPERA. 7, 7: 69-84.

Paqui, A y Poma, M (2013) "La pérdida de la identidad cultural del pueblo kichwa Saraguro en la comunidad Cisam, parroquia Nuevo Quito, cantón Paquisha". Trabajo de grado. Ciencias de la Educación con Mención en Educación Intercultural Bilingüe. Universidad de Cuenca.

Rivero, P y Martínez, V. (2016). "Cultura e identidad. Discusiones teóricas-epistemológicas para la comprensión de la contemporaneidad". Revista de Antropología Experimental. 16: 109-121. Acceso 5 de marzo de 2019. http://revistaselectronicas.ujaen.es/index.php/rae.

Rojas, M. (2004). «Identidad y cultura». Revista Educere, 8 (27): 489-496.

Salvador, S y Martínez, Y. (2017). Apuntes para una historia comunicativa de los Kichwa-Saraguro: de la kipa al teléfono móvil. Revista Andaluza de Antropología, 13, 1-24.

Seda, Gun E. 2012. "Attitudes of Primary School Teacher Candidates towards the Teaching Profession". Procedia - Social and Behavioral Sciences 46: 2922 - 2926. Acceso 2 de marzo de 2019. https://core.ac.uk, download, pdf.

Serón, J. (2019). Proyecto: «Conocer y reflexionar para llegar más lejos" Fase: Diagnóstico, Bloque: Cómo nos vemos (No. BOOK-2019-036). Universidad de Zaragoza.

UNESCO. (2005). EFA Global Monitoring Report. Informe. Paris. http://www.unesco.org/education/gmr_download/chapter1.pdf. 